\title{
Importance of Systematic Reviews in Implant Dentistry
}

\author{
Bruno Chrcanovic
}

International Journal of Prosthodontics and Restorative Dentistry (2019): 10.5005/jp-journals-10019-1234

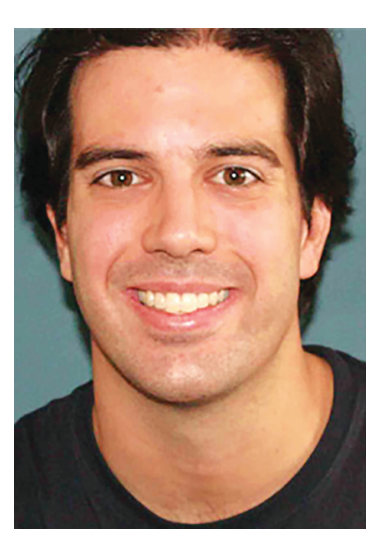

It is not unusual for the number of published studies in a specific area to run into hundreds or even thousands. Some of these individual studies may give unclear, confusing, or contradictory results, offering little insight into the problem at hand. When the studies are taken together within a review, a clearer and more consistent picture will emerge. Reviews have long been a part of the healthcare literature, in order to collate existing knowledge and publish summaries on specific topics. $^{1}$

There are two main types of reviews: the narrative and the systematic reviews. Narrative reviews are the traditional approach and usually do not include a section describing the methods used in the review. Though these narrative reviews are often conducted by people with expert knowledge on their field, this expertise and experience may bias the authors. The absence of a clear and objective method section leads to a number of methodological flaws, which can bias the author's conclusions. ${ }^{2}$ Narrative reviews are highly subjected to publication selection bias, as it can favor one treatment over another one based on the author's biased preferences to choose the studies. Systematic reviews use systematic methods and a reproducible search of a range of sources to collect secondary data. This is a major factor in distinguishing systematic reviews from traditional narrative reviews and helps minimize bias and therefore assists in achieving reliable estimates of effects. ${ }^{3}$ By weighing the trials and producing a pooled overall estimate, which is a quantitative rather than a qualitative assessment of the apparent strength of the results, systematic reviews may often conclude that the effect of a treatment is less impressive than a traditional narrative review would conclude. ${ }^{4}$

Performing a rigorous systematic review requires a careful scientific consideration at inception, meticulous, and laborious searching, as well as a considerable attention to methodological detail and analysis. Systematic reviews usually, but not necessarily, apply meta-analysis to examine a research question. ${ }^{1}$ Meta-analyses generally provide a greater degree of precision when compared with other study types because the estimate from the combined studies is based on a larger sample than any of the individual studies. This will provide narrower $95 \%$ confidence intervals than any study conducted on its own. ${ }^{5}$ When well conducted, they should give us the best possible estimate of any true effect. ${ }^{1}$ There is evidence that systematic reviews improve the reliability and accuracy of the conclusions; however, the results are rarely unequivocal and require a careful appraisal and interpretation: ${ }^{6}$
Department of Prosthodontics, Faculty of Odontology, Malmö University, Malmö, Sweden

Corresponding Author: Bruno Chrcanovic, Department of Prosthodontics, Faculty of Odontology, Malmö University, Malmö, Sweden, Phone: +46 406658520, e-mail: bruno.chrcanovic@mau.se

How to cite this article: Chrcanovic B. Importance of Systematic Reviews in Implant Dentistry. Int J Prosthodont Restor Dent 2019;9(2):36-37.

Source of support: Nil

Conflict of interest: None

Clinicians therefore need to integrate the results with clinical expertise and the patient's preferences.

Systematic reviews are needed to propose a future research agenda when the way forward may be unclear or the existing agendas have failed to address a clinical problem. Systematic reviews are most needed whenever there is a substantive question, several primary studies - sometimes even with disparate findingsand substantial uncertainty. ${ }^{1}$ This is no different in implant dentistry. There are still difficult or unusual clinical situations for which a true consensus has not been reached in the literature concerning the use of the dental implants. Some examples include the oral rehabilitation with dental implants of patients with bruxism or patients undergoing a chemotherapy. ${ }^{7}$ Also, other examples include the use of extra short implants, the installation of implants in the growing patient or in patients taking bisphosphonates, ${ }^{8}$ proton pump inhibitors, ${ }^{9}$ or selective serotonin reuptake inhibitors, among others. This is also true for many newer protocols, less invasive techniques, and procedures that do not follow conventional implant therapy are often performed in clinical practice without extensive literature support. ${ }^{10}$ A review of such scenarios is important because it provides information that can improve diagnostic accuracy, allowing dentists to make informed decisions and refine treatment plans to optimize clinical outcomes.

\section{References}

1. Hemingway $P$, Brereton N. What is a Systematic Review? Hayward Med Comm 2009;2:1-8.

2. Mulrow $C D$. The medical review article: state of the science. Ann Intern Med 1987;106(3):485-488. DOI: 10.7326/0003-4819-106-3-485.

3. Higgins JPT, Green S. Cochrane Collaboration. Cochrane handbook for systematic reviews of interventions. Chichester, England; Hoboken, NJ: Wiley-Blackwell; 2008.

4. Cipriani A, Geddes J. Comparison of systematic and narrative reviews: the example of the atypical antipsychotics. Epidemiol Psichiatr Soc 2003;12(3):146-153. DOI: 10.1017/S1121189X00002918.

(c) The Author(s). 2019 Open Access This article is distributed under the terms of the Creative Commons Attribution 4.0 International License (https://creativecommons. org/licenses/by-nc/4.0/), which permits unrestricted use, distribution, and non-commercial reproduction in any medium, provided you give appropriate credit to the original author(s) and the source, provide a link to the Creative Commons license, and indicate if changes were made. The Creative Commons Public Domain Dedication waiver (http://creativecommons.org/publicdomain/zero/1.0/) applies to the data made available in this article, unless otherwise stated. 
5. Kashi A, Saha S. Evidence-based techniques to assess the performance of dental implants. J Oral Implantol 2013;39(6):655-661. DOI: 10.1563/ AAID-JOI-D-10-00084.

6. Hopayian K. The need for caution in interpreting high-quality systematic reviews. BMJ 2001;323:681-684. DOI: 10.1136/ bmj.323.7314.681.

7. Chrcanovic BR, Albrektsson T, et al. Dental implants in patients receiving chemotherapy: A Meta-analysis. Implant Dent 2016;25(2): 261-271. DOI: 10.1097/ID.0000000000000388.
8. Chrcanovic BR, Albrektsson T, et al. Bisphosphonates and dental implants: A meta-analysis. Quintessence Int 2016;47(4):329-342. DOI: 10.3290/j.qi.a35523.

9. Chrcanovic BR, Kisch J, et al. Intake of proton pump inhibitors is associated with an increased risk of dental implant failure. Int J Oral Maxillofac Implants 2017;32(5):1097-1102. DOI: 10.11607/jomi.5662.

10. Aghaloo TL, Mardirosian M, et al. Controversies in implant surgery. Oral Maxillofac Surg Clin North Am 2017;29(4):525-535. DOI: 10.1016/ j.coms.2017.07.007. 